\title{
BOUNDARY BEHAVIOR OF THE QUASI-HYPERBOLIC METRIC
}

\author{
Nikolai Nikolov and Pascal J. Thomas
}

Bulgarian Academy of Sciences, Institute of Mathematics and Informatics Acad. G. Bonchev 8, 1113 Sofia, Bulgaria, and

State University of Library Studies and Information Technologies, Faculty of Information Sciences Shipchenski prohod 69A, 1574 Sofia, Bulgaria; nik@math.bas.bg

Institut de Mathématiques de Toulouse; UMR5219, Université de Toulouse; CNRS; UPS IMT

F-31062 Toulouse Cedex 9, France; pascal.thomas@math.univ-toulouse.fr

\begin{abstract}
The precise behavior of the quasi-hyperbolic metric near a $\mathcal{C}^{1,1}$-smooth part of the boundary of a domain in $\mathbf{R}^{n}$ is obtained.
\end{abstract}

\section{Introduction and results}

Let $D$ be a proper subdomain of $\mathbf{R}^{n}$. Define the quasi-hyperbolic metric of $D$ by

$$
h_{D}(a, b)=\inf _{\gamma} \int_{\gamma} \frac{\|d u\|}{d_{D}(u)}, \quad a, b \in D
$$

where $\|\cdot\|$ is the Euclidean norm, $d_{D}=\operatorname{dist}(\cdot, \partial D)$ and the infimum is taken over all rectifiable curves $\gamma$ in $D$ joining $a$ to $b$. By [5, Lemma 1], the infimum is attained, and any extremal curve is called quasi-hyperbolic geodesic (for short, geodesic). It turns out that the geodesics are $\mathcal{C}^{1,1}$-smooth (see [8, Corollary 4.8]). The quasi-hyperbolic metric arises in the theory of quasi-conformal maps.

This paper is devoted to the boundary behavior of $h_{D}$. First, we point out the following general lower bound.

Proposition 1. [4, Lemma 2.6] If $D$ is a proper subdomain of $\mathbf{R}^{n}$, then

$$
h_{D}(a, b) \geq 2 \log \frac{d_{D}(a)+d_{D}(b)+\|a-b\|}{2 \sqrt{d_{D}(a) d_{D}(b)}}, \quad a, b \in D .
$$

Observe that equality occurs if $n=1$ (then $D$ is an open interval or ray).

From now, we assume that $n \geq 2$. Throughout the paper, we will say that $\zeta$ is a $\mathcal{C}^{\alpha}$-smooth boundary point of $D$ if and only if it admits a neighborhood in which $\partial D$ is $\mathcal{C}^{\alpha}$-smooth.

Recall that a $\mathcal{C}^{1}$-smooth boundary point $\zeta$ of a domain $D$ in $\mathbf{R}^{n}$ is said to be Dinismooth if the inner unit normal vector $n$ to $\partial D$ near $\zeta$ is a Dini-continuous function. This means that there exists a neighborhood $U$ of $\zeta$ such that $\int_{0}^{1} \frac{\omega(t)}{t} d t<+\infty$, where

$$
\omega(t)=\omega(n, \partial D \cap U, t):=\sup \left\{\left\|n_{x}-n_{y}\right\|:\|x-y\|<t, x, y \in \partial D \cap U\right\}
$$

is the respective modulus of continuity.

If $\int_{0}^{1} \omega(t) \frac{\log t}{t} d t>-\infty$, then the point $\zeta$ is called log-Dini smooth.

https://doi.org/10.5186/aasfm.2018.4318

2010 Mathematics Subject Classification: Primary 51M10, 32F45, 30C65.

Key words: Quasi-hyperbolic metric, Kobayashi distance.

This paper was started while the first-named author was visiting the Paul Sabatier University, Toulouse in November 2015. 
The following relations between different notions of smoothness are clear: $\mathcal{C}^{1, \varepsilon} \Longrightarrow$ $\log$-Dini $\Longrightarrow$ Dini $\Longrightarrow \mathcal{C}^{1}$.

Theorem 2. [9, Theorem 7] Let $\zeta$ be a Dini-smooth boundary point of a domain $D$ in $\mathbf{R}^{n}$. Then for any constant $c>1+\frac{\sqrt{2}}{2}$ there exists a neighborhood $U$ of $\zeta$ such that

$$
h_{D}(a, b) \leq 2 \log \left(1+\frac{c\|a-b\|}{\sqrt{d_{D}(a) d_{D}(b)}}\right), \quad a, b \in D \cap U .
$$

Since $h_{D}$ is an inner metric, we get an upper bound of $h_{D}$, similar to the lower bound from Proposition 1.

Corollary 3. [9, Corollary 8] Let $D$ be a Dini-smooth bounded domain in $\mathbf{R}^{n}$. Then there exists a constant $c>0$ such that

$$
h_{D}(a, b) \leq 2 \log \left(1+\frac{c\|a-b\|}{\sqrt{d_{D}(a) d_{D}(b)}}\right), \quad a, b \in D .
$$

Set now

$$
\begin{aligned}
s_{D}(a, b) & =2 \sinh ^{-1} \frac{\|a-b\|}{2 \sqrt{d_{D}(a) d_{D}(b)}} \\
& =2 \log \frac{\|a-b\|+\sqrt{\|a-b\|^{2}+4 d_{D}(a) d_{D}(b)}}{2 \sqrt{d_{D}(a) d_{D}(b)}}, \quad a, b \in D .
\end{aligned}
$$

Note that $h_{D}=s_{D}$ if $D$ is a half-space in $\mathbf{R}^{n}$ (cf. [12, (2.8)]).

The following sharp result holds in the $\mathcal{C}^{1}$-smooth case.

Proposition 4. $[9$, Proposition $6(\mathrm{a})]$ If $\zeta$ is a $\mathcal{C}^{1}$-smooth boundary point of a domain $D$ in $\mathbf{R}^{n}$, then

$$
\lim _{\substack{a, b \rightarrow \zeta \\ a \neq b}} \frac{h_{D}(a, b)}{s_{D}(a, b)}=1 .
$$

Since the proof of this proposition is not long, we shall include it for completeness.

Corollary 5. [9, Proposition 6(b) and p. 3] If $D$ is a $\mathcal{C}^{1}$-smooth bounded domain in $\mathbf{R}^{n}$, then

is a continuous function on $\mathbf{R}^{n} \times \mathbf{R}^{n}$.

$$
q_{D}(a, b)= \begin{cases}\frac{h_{D}(a, b)}{s_{D}(a, b)}, & a, b \in D, a \neq b \\ 1, & \text { otherwise, }\end{cases}
$$

The main goal of this paper is to prove the following result related to Proposition 4 .

Theorem 6. If $\zeta$ is a $\mathcal{C}^{1,1}$-smooth boundary point of a domain $D$ in $\mathbf{R}^{n}$, then

$$
\lim _{a, b \rightarrow \zeta}\left(h_{D}(a, b)-s_{D}(a, b)\right)=0 .
$$

Note that Theorem 6 and Proposition 4 say the same only if $s_{D}$ and $1 / s_{D}$ are bounded.

The assumption about regularity in Theorem 6 can be weakened in the plane.

Proposition 7. If $\zeta$ is a log-Dini smooth boundary point of a domain $D$ in $\mathbf{R}^{2}$, then

$$
\lim _{a, b \rightarrow \zeta}\left(h_{D}(a, b)-s_{D}(a, b)\right)=0 .
$$


The above results imply the following optimal version of Theorem 2 .

Corollary 8. Let $\zeta$ be a $\mathcal{C}^{1,1}$-smooth boundary point of a domain $D$ in $\mathbf{R}^{n}$ or $\zeta$ be a log-Dini smooth boundary point of a domain $D$ in $\mathbf{R}^{2}$. Then for any constant $c>1$ there exists a neighborhood $U$ of $\zeta$ such that

$$
h_{D}(a, b) \leq 2 \log \left(1+\frac{c\|a-b\|}{\sqrt{d_{D}(a) d_{D}(b)}}\right), \quad a, b \in D \cap U .
$$

The rest of the paper is organized as follows: Section 2 contains the proofs of Propositions 4, 7 and Corollary 8. Section 3 contains the proof of Theorem 6. It should be mentioned that the three proofs use different flattening maps. Section 4 contains the proof of a result analogous to Corollary 8 for the Kobayashi distance.

\section{Proofs of Propositions 4, 7 and Corollary 8}

Proof of Proposition 4. After translation and rotation, we may assume that $\zeta=0$ and that there is a neighborhood $U$ of 0 such that

$$
D^{\prime}:=D \cap U=\left\{x \in U: r(x):=x_{1}+f\left(x^{\prime}\right)>0\right\},
$$

where points of $\mathbf{R}^{n}$ are denoted by $x=\left(x_{1}, x^{\prime}\right)$, with $x^{\prime} \in \mathbf{R}^{n-1}$, and $f$ is a $\mathcal{C}^{1}$-smooth function in $\mathbf{R}^{n}$ with $f(0)=0$ and $\nabla f(0)=0$.

Let $c>1$ and $\theta(x)=\left(r(x), x^{\prime}\right)$. We may shrink $U$ such that

$$
c^{-1}\|x-y\| \leq\|\theta(x)-\theta(y)\| \leq c\|x-y\|, \quad x, y \in U .
$$

Choose now a neighborhood $V \subset U$ of 0 such that $d_{D^{\prime}}=d_{D}$ on $D \cap V$. The regularity of $D$ implies that it is a uniform domain near $\zeta$ in the sense of [5]. Using, for example, [5, Corollary 2], one can find a neighborhood $W \subset V$ of 0 such that any geodesic joining points in $\tilde{D}=D \cap W$ is contained in $D \cap V$. Then $h_{D}=h_{D^{\prime}}$ on $\tilde{D}^{2}$.

Set $\mathbf{R}_{+}^{n}=\left\{x \in \mathbf{R}^{n}: x_{1}>0\right\}$. Using the above arguments, we may shrink $W$ such that $h_{\mathbf{R}_{+}^{n}}=h_{\theta\left(D^{\prime}\right)}$ on $(\theta(\tilde{D}))^{2}$.

On the other hand, (1) implies that (cf. [12, Exercise 3.17])

$$
c^{-2} h_{D^{\prime}}(z, w) \leq h_{\theta\left(D^{\prime}\right)}(\theta(z), \theta(w)) \leq c^{2} h_{D^{\prime}}(z, w), \quad z, w \in D^{\prime} .
$$

Let $z, w \in \tilde{D}$. Then

$$
c^{-2} h_{D}(z, w) \leq h_{\mathbf{R}_{+}^{n}}(\theta(z), \theta(w)) \leq c^{2} h_{D}(z, w) .
$$

Using (1) again, we get that

$$
\begin{aligned}
h_{\mathbf{R}_{+}^{n}}(\theta(z), \theta(w)) & =2 \sinh ^{-1} \frac{\|\theta(z)-\theta(w)\|}{2 \sqrt{r_{D}(z) r_{D}(w)}} \leq 2 \sinh ^{-1} \frac{c^{2}\|z-w\|}{2 \sqrt{d_{D}(z) d_{D}(w)}} \\
& \leq c^{2} s_{D}(z, w) .
\end{aligned}
$$

We obtain in the same way that

$$
h_{\mathbf{R}_{+}^{n}}(\theta(z), \theta(w)) \geq c^{-2} s_{D}(z, w) .
$$

So

$$
c^{-4} h_{D}(z, w) \leq s_{D}(z, w) \leq c^{4} h_{D}(z, w)
$$

which implies the desired result.

Proof of Proposition 7. We may find a neighborhood $U$ of $\zeta$ such that $D \cap U$ is a bounded simply connected log-Dini smooth domain. Using an argument from the previous proof, we may replace $D$ by $D \cap U$. 
The Kellogg-Warschawski theorem (cf. [11, Theorem 3.5]) implies that there exists a conformal map $\tilde{f}$ from the unit disc $\mathbf{D}$ to $D$ which extends to a $\mathcal{C}^{1}$-diffeomorphism between $\overline{\mathbf{D}}$ to $\bar{D}$ such that $\tilde{f}(\zeta)=1$ and

$$
\left|\tilde{f}^{\prime}(z)-\tilde{f}^{\prime}(w)\right| \leq \tilde{\omega}^{*}(|z-w|), \quad z, w \in \mathbf{D}
$$

where $\tilde{\omega}^{*}(s)=\int_{0}^{s} \frac{\tilde{\omega}(t)}{t} d t+s \int_{s}^{+\infty} \frac{\tilde{\omega}(t)}{t^{2}} d t(s \geq 0)$ and $\tilde{\omega}: \mathbf{R}^{+} \rightarrow \mathbf{R}^{+}$is a bounded continuous function with $\int_{0}^{1} \tilde{\omega}(t) \frac{\log t}{t} d t>-\infty$.

Then $f(z)=\tilde{f}\left(\frac{1-z}{1+z}\right)$ maps conformally $\mathbf{R}_{+}^{2}$ onto $D$ and

$$
\left|f^{\prime}(z)-f^{\prime}(w)\right| \leq \omega^{*}(|z-w|), \quad z, w \in G=\mathbf{R}_{+}^{2} \cap \mathbf{D}
$$

where $\omega^{*}$ is defined from $\omega$ in the same way as $\tilde{\omega}^{*}$.

The equality

$$
f(w)-f(z)-f^{\prime}(z)(w-z)=(w-z) \int_{0}^{1}\left(f^{\prime}(z+t(w-z))-f^{\prime}(z)\right) d t
$$

implies that

$$
\left|f(w)-f(z)-f^{\prime}(z)(w-z)\right| \leq|w-z| \omega^{*}(|w-z|)
$$

(since $\omega^{*}$ is an increasing function). It follows that

$$
\left|d_{D}(f(z))-\right| f^{\prime}(z)\left|d_{\mathbf{R}_{+}^{2}}(z)\right| \leq d_{\mathbf{R}_{+}^{2}}(z) \omega^{*}\left(d_{\mathbf{R}_{+}^{2}}(z)\right), \quad z \in G .
$$

Since $D$ is a uniform domain, there exists a neighborhood $V$ of $\zeta$ such that any geodesic $\gamma$ joining points $a=f(\alpha)$ and $b=f(\beta)$ in $D \cap V$ is contained in $f(G)$. It follows by (2) that one may find a constant $C>0$ (independent of $a$ and $b$ ) such that

$$
h_{\mathbf{R}_{+}^{2}}(\alpha, \beta) \leq \int_{f^{-1} \circ \gamma} \frac{|d u|}{d_{\mathbf{R}_{+}^{2}}(u)} \leq \int_{\gamma} \frac{|d v|}{d_{D}(v)}+C \int_{\gamma} \frac{\omega^{*}\left(d_{D}(v)\right)}{d_{D}(v)}|d v| .
$$

The first summand is equal to $h_{D}(a, b)$.

We claim that the second summand tends to 0 as $a, b \rightarrow \zeta$. Indeed, denote by $t$ the natural parameter of $\gamma$ by arc length and by $l=l(\gamma)$ the Euclidean length of $\gamma$. Since $D$ is a uniform domain, then [5, Corollary 2] provides a constant $c>0$ (independent of $a$ and $b$ ) such that $c \cdot l \leq|a-b|$ and $d_{D}(\gamma(t)) \geq c \cdot \max \{t, l-t\}$. Using that $\frac{\omega^{*}(s)}{s}$ is a decreasing function, we get

$$
\int_{\gamma} \frac{\omega^{*}\left(d_{D}(v)\right)}{d_{D}(v)}|d v| \leq \frac{2}{c} \int_{0}^{c l / 2} \frac{\omega^{*}(t)}{t} d t .
$$

It is easy to check the log-Dini condition for $\omega$ is is equivalent to the fact that the last integral tends to 0 as $l \rightarrow 0$ which implies our claim.

Hence

$$
\liminf _{a, b \rightarrow \zeta}\left(h_{D}(a, b)-h_{\mathbf{R}_{+}^{2}}(\alpha, \beta)\right) \geq 0 .
$$

The opposite inequality

$$
\limsup _{a, b \rightarrow \zeta}\left(h_{D}(a, b)-h_{\mathbf{R}_{+}^{2}}(\alpha, \beta)\right) \leq 0
$$

follows in the same way by taking the geodesic joining $\alpha$ and $\beta$.

Using (2), we have that

$$
\lim _{\substack{a, b \rightarrow \zeta \\ a \neq b}} \frac{|a-b|}{2 \sqrt{d_{D}(a) d_{D}(b)}} \cdot \frac{2 \sqrt{d_{\mathbf{R}_{+}^{2}}(\alpha) d_{\mathbf{R}_{+}^{2}}(\beta)}}{|\alpha-\beta|}=1 .
$$


Since $h_{\mathbf{R}_{+}^{2}}=s_{\mathbf{R}_{+}^{2}}$ and $\sinh ^{-1} q t<\log q+\sinh ^{-1} t$ for $q>1, t>0$, then

$$
\lim _{a, b \rightarrow \zeta}\left(s_{D}(a, b)-h_{\mathbf{R}_{+}^{2}}(\alpha, \beta)\right)=0
$$

which completes the proof.

Proof of Corollary 8. We may assume that $c=2 c^{\prime}-1 \in(1,3]$. By Proposition 4, Theorem 6 and Proposition 7, one may find a neighborhood $U$ of $\zeta$ such that for $a, b \in D \cap U$,

$$
h_{D}(a, b) \leq c^{\prime} s_{D}(a, b), \quad h_{D}(a, b) \leq s_{D}(a, b)+\log c^{\prime} .
$$

Then the result follows by the inequalities $\sinh ^{-1} \frac{t}{2}<\log (1+t)(t>0),(1+t)^{c^{\prime}}<$ $1+c t(0<t<1)$ and $c^{\prime}(1+t)<1+c t(t>1)$.

\section{Proof of Theorem 6}

Theorem 6 will follow from Propositions 9 and 11 below.

For convenience, we assume that $D$ is a domain in $\mathbf{R}^{n+1}(n \geq 1)$. We first localize the problem. We choose local coordinates so that $\zeta=0$ and $T_{0} \partial D=\{0\} \times \mathbf{R}^{n}$. Denote points in $\mathbf{R}^{n+1}$ by $\bar{x}=\left(x_{0}, x\right) \in \mathbf{R} \times \mathbf{R}^{n}$. We also write $\mathbf{R}_{+}^{n+1}=\{\bar{x} \in$ $\left.\mathbf{R}^{n+1}: x_{0}>0\right\}$.

There are a ball $\mathcal{U} \subset \mathbf{R}^{n+1}$ centered at $(0,0)$ and a function $f \in \mathcal{C}^{1,1}\left(\mathcal{U} \cap \mathbf{R}^{n}, \mathbf{R}\right)$ such that $f(0)=0$ and $D f(0)=0$ and

$$
D \cap \mathcal{U}=\left\{\bar{x} \in \mathcal{U}: x_{0}>f(x)\right\} .
$$

By shrinking the radius of $\mathcal{U}$ further we may assume that the projection which to $\bar{x} \in \mathcal{U} \cap D$ associates $\pi(\bar{x})$, the closest point in $\partial D$ is well-defined, and that $\mathcal{U} \subset$ $\pi^{-1}(\mathcal{U} \cap D)$ (see [1, Lemma 4.11], or the proof of Lemma 10 (1) below).

Proposition 9. $\liminf _{a, b \rightarrow 0}\left(h_{D}(a, b)-s_{D}(a, b)\right) \geq 0$.

We can define a map $\varphi$ on $\mathcal{U}$ by

$$
\varphi(\bar{x})=(f(x), x)+x_{0} n_{x},
$$

where $n_{x}$ is the inward unit normal to $\partial D$ at the point $(f(x), x)$.

Lemma 10. (1) There exists a ball $\mathcal{U}_{0} \subset \mathcal{U}$ centered at 0 such that $\left.\varphi\right|_{\mathcal{U}_{0}}$ is a bilipschitz homeomorphism and for any $\bar{x} \in \mathcal{U}_{0} \cap \mathbf{R}_{+}^{n+1}$,

$$
d_{D} \varphi(\bar{x})=\|\varphi(\bar{x})-(f(x), x)\|=x_{0} .
$$

(2) Furthermore, if $f \in \mathcal{C}^{\alpha}\left(\mathcal{U} \cap \mathbf{R}^{n}, \mathbf{R}\right)$, for some $\alpha \geq 2$, then $\left.\varphi\right|_{\mathcal{U}_{0}}$ is a $\mathcal{C}^{\alpha-1}$ diffeomorphism, and there exists a ball $\mathcal{U}_{1} \subset \mathcal{U}_{0}$ centered at 0 and a constant $C>0$ such that for any $\bar{x} \in \mathcal{U}_{1} \cap \mathbf{R}_{+}^{n+1}$ and any vector $v \in \mathbf{R}^{n+1}$,

$$
\|D \varphi(\bar{x}) \cdot v\| \geq\left(1-C x_{0}\right)\|v\|,
$$

where $D \varphi(\bar{x})$ stands for the differential of $\varphi$ taken at the point $\bar{x}$.

(3) In the general case where $f \in \mathcal{C}^{1,1}\left(\mathcal{U} \cap \mathbf{R}^{n}, \mathbf{R}\right)$, then there exists a $C>0$ such that for any $\mathcal{C}^{1}$ curve $\gamma:\left[t_{1}, t_{2}\right] \longrightarrow \mathcal{U}_{1} \cap \mathbf{R}_{+}^{n+1}, \varphi \circ \gamma$ is rectifiable and for any $F \in \mathcal{C}\left(\left[t_{1}, t_{2}\right], \mathbf{R}_{+}\right)$,

$$
\int_{t_{1}}^{t_{2}} F(t)|d \varphi \circ \gamma(t)| \geq \int_{t_{1}}^{t_{2}} F(t)|d \gamma(t)|-C \int_{t_{1}}^{t_{2}} F(t) d_{D}(\gamma(t))|d \gamma(t)| .
$$


Proof. Part (1) of the lemma is classical (see [1, Theorem 4.8]). The main point is to prove that the domain has positive reach, that is to say that there exists $\delta>0$ such that if $x \in D$ and $d_{D}(x)<\delta$, then this distance is attained at a single point, which will be the intersection of $\partial D$ and the unique normal line to it containing $x$ (see [1]). In other words, for $x \in \mathcal{U}$ well chosen and $x_{0}<\delta, \varphi$ is one-to-one.

We quickly recall the proof. Suppose $\left\|\nabla f(x)-\nabla f\left(x^{\prime}\right)\right\| \leq L\left\|x-x^{\prime}\right\|$ for $(0, x)$, $\left(0, x^{\prime}\right) \in \mathcal{U}_{1}$, then, taking without loss of generality the projection to $\partial D$ to be $(0,0)$, for some $\theta \in(0,1)$,

$$
\begin{aligned}
\left\|\left(y_{0}, 0\right)-(f(x), x)\right\|^{2} & =y_{0}^{2}-2 y_{0} \nabla f(\theta x) \cdot x+f(x)^{2}+\|x\|^{2} \\
& \geq y_{0}^{2}+\|x\|^{2}-2 y_{0} L\|x\|^{2}>y_{0}^{2}
\end{aligned}
$$

for $y_{0}<1 / 2 L$ and $x \neq 0$.

Notice that a lemma in [6, Appendix], explained in detail in [7], shows that even though $n_{x}$ can only be expected to be continuous with bounded derivatives, and in general of class $\mathcal{C}^{\alpha-1}$ when $\varphi \in \mathcal{C}^{\alpha}$, the function $\bar{x} \mapsto d_{D}(\bar{x})$ has the same regularity as $\varphi$.

We now prove part (2). Let $\left(e_{0}, e_{1}, \ldots, e_{n}\right)$ be the standard basis of $\mathbf{R}^{n+1}$. Let $\tilde{e}_{j}=\frac{\partial f}{\partial x_{j}}(x) e_{0}+e_{j}$, for $1 \leq j \leq n$. They form a basis of the tangent space to $\partial D$ at $(x, f(x))$ and $\left\langle n_{x}, \tilde{e}_{j}\right\rangle=0$ for $1 \leq j \leq n$. Then $D \varphi(\bar{x}) \cdot e_{0}=n_{x}$, and $D \varphi(\bar{x}) \cdot e_{j}=\tilde{e}_{j}+x_{0} \frac{\partial n_{x}}{\partial x_{j}}$, for $1 \leq j \leq n$.

Given $v=\sum_{0}^{n} v_{j} e_{j}$,

$$
D \varphi(\bar{x}) \cdot v=\left(v_{0} n_{x}+\sum_{1}^{n} v_{j} \tilde{e}_{j}\right)+x_{0} \sum_{1}^{n} v_{j} \frac{\partial n_{x}}{\partial x_{j}}=: V_{1}+V_{0}
$$

Clearly, $\left\|V_{0}\right\|=O\left(x_{0}\right)\|v\|$. By the orthogonality of $n_{x}$ to the tangent space,

$$
\begin{aligned}
\left\|V_{1}\right\|^{2} & =v_{0}^{2}+\left\|\sum_{1}^{n} v_{j} \tilde{e}_{j}\right\|^{2}=v_{0}^{2}+\left\|\mid \sum_{1}^{n} v_{j} e_{j}+\left(\sum_{1}^{n} v_{j} \frac{\partial f}{\partial x_{j}}(x)\right) e_{0}\right\|^{2} \\
& =v_{0}^{2}+\sum_{1}^{n} v_{j}^{2}+\left|\sum_{1}^{n} v_{j} \frac{\partial f}{\partial x_{j}}(x)\right|^{2} \geq\|v\|^{2} .
\end{aligned}
$$

In the case where $f \in \mathcal{C}^{1,1}$, then $\varphi \circ \gamma$ is only a Lipschitz map. By Rademacher's theorem (see e.g. [2, Theorem 3.1.6]), it is almost everywhere differentiable and the fundamental theorem of calculus holds. We then perform the same calculation as in case (2), where the integrands are defined a.e.

Proof of Proposition 9. Using Lemma 10, the proof repeats the second part of the proof of Proposition 7. Suppose that $\zeta=0$ and that the domain $D$ is given by a local representation as above. We may assume that the points $a, b \in D$ are in a small enough neighborhood of 0 so that the geodesic $\gamma$ which joins them is entirely contained in the range of invertibility of $\varphi$ and Lemma 10 holds; we write $a=\varphi(\bar{\alpha})$, $b=\varphi(\bar{\beta}), \gamma=\varphi(\tilde{\gamma})$, where $\tilde{\gamma}$ is an arc in $\mathbf{R}_{+}^{n+1}$. Then

$$
h_{D}(a, b)=\int_{\gamma} \frac{\|d u\|}{d_{D}(u)} \geq \int_{\tilde{\gamma}} \frac{\|d v\|}{d_{\mathbf{R}_{+}^{n+1}}(v)}-C \cdot l(\tilde{\gamma}) \geq h_{\mathbf{R}_{+}^{n+1}}(\bar{\alpha}, \bar{\beta})-C^{\prime}\|\bar{\alpha}-\bar{\beta}\|,
$$


where $C^{\prime}>0$ is a constant independent of $a$ and $b$. Note that $h_{\mathbf{R}_{+}^{n+1}}=s_{\mathbf{R}_{+}^{n+1}}$. Since the differential of $\varphi$ at $\bar{x}$ tends to the identity as $x \rightarrow 0$, it follows that

$$
\lim _{a, b \rightarrow \zeta}\left(s_{\mathbf{R}_{+}^{n+1}}(\bar{\alpha}, \bar{\beta})-s_{D}(a, b)\right)=0
$$

which completes the proof.

Proposition 11. $\lim \sup _{a, b \rightarrow 0}\left(h_{D}(a, b)-s_{D}(a, b)\right) \leq 0$.

The proof is similar to that of Proposition 9, using a modification of the map $\varphi$ which depends on $a$ and $b$.

Proof. We again assume that $a, b \in D$, and the geodesic connecting them, all lie in a neighborhood of $\zeta$ small enough so that any point in it has a unique closest point on $\partial D$. Let $a^{\prime}, b^{\prime}$ be the respective closest points. We take new coordinates (and obtain a new function $f$ ) so that $a^{\prime}=0$ (instead of $\zeta=0$ as in the proof of Proposition 9) and

$$
D \cap \mathcal{U}=\left\{\bar{x} \in \mathcal{U}: x_{0}>f\left(x_{1}, \ldots, x_{n}\right)\right\} .
$$

We may also assume that $b_{2}^{\prime}=\cdots=b_{n}^{\prime}=0$. Shrinking the radius $r$ of $\mathcal{U}$, we may replace $x_{1}$ by $\sigma_{1}\left(x_{1}\right)$ such that for $\sigma=\left(f\left(\sigma_{1}, 0, \ldots, 0\right), \sigma_{1}, 0, \ldots, 0\right)$ one has $\left\|\sigma^{\prime}\right\|=1$ (in other words, $\sigma$ is parametrized by arc length). Note that $r$ can be chosen independently of $a$ and $b$. Let $\ell$ be the length of the curve $\sigma$ from $a^{\prime}$ to $b^{\prime}$, so that $\sigma(0)=a^{\prime}, \sigma(\ell)=b^{\prime}$.

Consider the map $\varphi$ from $\mathbf{R}_{+}^{2}$ (near 0 ) to $D$ defined by

$$
\varphi\left(x_{0}, x_{1}\right)=\sigma\left(x_{1}\right)+x_{0} n_{\sigma\left(x_{1}\right)},
$$

where $n_{\sigma\left(x_{1}\right)}$ is the inward unit normal to $\partial D$ at the point $\sigma\left(x_{1}\right)$. Then $d_{D}(\varphi(\bar{x}))=x_{0}$ if $x_{0}$ is small enough, and if $\alpha=\left(d_{D}(a), 0\right)$ and $\beta=\left(d_{D}(b), \ell\right)$, we have $\varphi(\alpha)=a$, $\varphi(\beta)=b$.

Lemma 12. There exist a neighborhood $U$ of $\zeta$, a neighborhood $V$ of 0 and a constant $C>0$ such that for any $a, b \in D \cap U$ and $\bar{x} \in \mathbf{R}_{+}^{2} \cap V$ and any vector $v \in \mathbf{R}^{2}$, then $\alpha, \beta \in V$ and

$$
\|D \varphi(\bar{x}) \cdot v\| \leq\left(1+C x_{0}\right)\|v\| .
$$

Proof. As in the proof of Lemma 10 (2), in the $\mathcal{C}^{2}$-smooth case,

$$
D \varphi(\bar{x}) \cdot e_{0}=n_{\sigma\left(x_{1}\right)}, \quad D \varphi(\bar{x}) \cdot e_{1}=\sigma^{\prime}\left(x_{1}\right)+x_{0} \frac{\partial n_{\sigma\left(x_{1}\right)}}{\partial x_{1}} .
$$

Because $\left\|\sigma^{\prime}\right\|=1$ and is tangent to $\partial D,\left(\sigma^{\prime}(x), n_{x}\right)$ form an orthonormal system, so that $D \psi(\bar{x})$ differs from a linear isometric embedding by a term bounded by $\left\|\frac{\partial n_{\sigma\left(x_{1}\right)}}{\partial x_{1}}\right\| x_{0}$.

Geometric considerations show that $\left\|\frac{\partial n_{\sigma\left(x_{1}\right)}}{\partial x_{1}}\right\| \leq \frac{1}{R}$ whenever there exist two balls $B_{1}, B_{2}$ of radius $R$, tangent to each side of $\partial D$ at $\sigma\left(x_{1}\right)$. The argument in the proof of Lemma 10 (1) shows there exists $\delta>0$ (depending only on the neighborhood $\mathcal{U}_{0}$ mentioned in that lemma) such that there exist two such balls of radius $\delta$ at each point in $\mathcal{U}_{0} \cap \partial D$.

As in the proof of Lemma $10(3)$, the $\mathcal{C}^{1,1}$-smooth case follows by applying Rademacher's theorem.

The proof of Proposition 11 can be finished similarly to that of Proposition 9. Let $\gamma$ be the geodesic joining $\alpha$ to $\beta$ in $\mathbf{R}_{+}^{2}$. Let $U, V$ be as in Lemma 12. Shrinking 
$V$ if needed so that $\varphi(V) \subset U$, we have $d_{D}(\varphi(u))=d_{\mathbf{R}_{+}^{2}}(u)$ for any $u \in \gamma$. Since $\varphi \circ \gamma$ is a curve joining $a$ to $b$ in $D$, using Lemma 12, we get

$$
\begin{aligned}
h_{D}(a, b) & \leq \int_{0}^{\ell} \frac{\left\|D \varphi(\gamma(t)) \cdot \gamma^{\prime}(t)\right\|}{d_{D}(\varphi \circ \gamma(t))} d t \leq h_{\mathbf{R}_{+}^{2}}(\alpha, \beta)+C l(\gamma) \\
& <s_{\mathbf{R}_{+}^{2}}(\alpha, \beta)+C \pi\|\alpha-\beta\|
\end{aligned}
$$

(here $\pi$ is the Ludolphine number, not the projection). The differential of $\varphi$ is close to a linear isometric embedding of $\mathbf{R}^{2}$ in $\mathbf{R}^{n+1}$ and hence we have the asymptotic relation (3) and

$$
\lim _{a, b \rightarrow \zeta}\left(s_{\mathbf{R}_{+}^{2}}(\alpha, \beta)-s_{D}(a, b)\right)=0,
$$

which completes the proof.

\section{An upper estimate for the Kobayashi distance}

Let $D$ be a domain in $\mathbf{C}^{n}$. The Kobayashi (pseudo) distance $k_{D}$ is obtained from the Lempert function

$$
l_{D}(a, b)=\inf \left\{\tanh ^{-1}|\alpha|: \exists \varphi \in \mathcal{O}(\mathbf{D}, D) \text { with } \varphi(0)=a, \varphi(\alpha)=b\right\}, \quad a, b \in D .
$$

The Lempert function does not always satisfy the triangle inequality, but setting

$$
k_{D}(a, b):=\inf \left\{\sum_{j=0}^{m-1} l_{D}\left(a_{j}, a_{j+1}\right): a_{j} \in D, a_{0}=a, a_{m}=b, m \geq 1\right\},
$$

one does obtain a (pseudo) distance, which is the largest that is dominated by $l_{D}$.

Recall that $k_{D}$ is the integrated form of the Kobayashi (pseudo) metric $\kappa_{D}(a ; X)=\inf \left\{|\alpha|: \exists \varphi \in \mathcal{O}(\mathbf{D}, D)\right.$ with $\left.\varphi(0)=a, \alpha \varphi^{\prime}(0)=X\right\}, \quad a \in D, X \in \mathbf{C}^{n}$.

Note that Theorem 2 and Proposition 7 (even in the Dini-smooth case) hold for $2 k_{D}$ instead of $h_{D}$ (see [9, Theorem 7] and [10, Proposition 6]). Moreover, the following result corresponds to Proposition 4.

Proposition 13. [9, Proposition 5(a)] If $\zeta$ is a $\mathcal{C}^{1}$-smooth boundary point of a domain $D$ in $\mathbf{C}^{n}$, then

$$
\limsup _{\substack{a, b \rightarrow \zeta \\ a \neq b}} \frac{2 k_{D}(a, b)}{h_{D}(a, b)} \leq 1
$$

It turns out that Corollary 8 also holds for $2 k_{D}$ instead of $h_{D}$. This gives the optimal version of [3, Proposition 2.5] in the $\mathcal{C}^{1,1}$-smooth case.

Proposition 14. Let $\zeta$ be a $\mathcal{C}^{1,1}$-smooth boundary point of a domain $D$ in $\mathbf{C}^{n}$ or $\zeta$ be a $\log$-Dini smooth boundary point of a domain $D$ in $\mathbf{C}$. Then for any constant $c>1$ there exists a neighborhood $U$ of $\zeta$ such that

$$
k_{D}(a, b) \leq \log \left(1+\frac{c\|a-b\|}{\sqrt{d_{D}(a) d_{D}(b)}}\right), \quad a, b \in D \cap U .
$$

Proof. Having in mind Corollary 8 , it is enough to show that

$$
\limsup _{\substack{a, b \rightarrow \zeta \\ a \neq b}} \frac{2 k_{D}(a, b)-h_{D}(a, b)}{\|a-b\|}<+\infty .
$$

Since $k_{D}$ is the integrated form of $\kappa_{D}$ and the lengths of the quasi-hyperbolic geodesics joining points in $D$ near $\zeta$ are bounded up to a multiplicative constant by 
the Euclidean distances between the points, the last inequality will be a consequence of the following one:

$$
\limsup _{\substack{a \rightarrow \zeta \\\|X\|=1}}\left(2 \kappa_{D}(a ; X)-\frac{1}{d_{D}(a)}\right)<+\infty .
$$

To see this, note that there exists an $r>0$ such that any $a \in D$ near $\zeta$ is contained in a (unique) ball $\mathbf{B}_{n}(\tilde{a}, r) \subset D$ with $r-\|a-\tilde{a}\|=d_{D}(a)$ (the inner ball condition). It remains to use that for such an $a$ and $\|X\|=1$ one has that

$$
\kappa_{D}(a ; X) \leq \kappa_{\mathbf{B}_{n}(\tilde{a}, r)}(a ; X) \leq \frac{r}{r^{2}-\|a-\tilde{a}\|^{2}}<\frac{1}{2 d_{D}(a)}+\frac{1}{4 r} .
$$

\section{References}

[1] Federer, H.: Curvature measures. - Trans. Amer. Math. Soc. 93, 1959, 418-491.

[2] Federer, H.: Geometric measure theory. - Grundlehren Math. Wiss. 153, Springer, Berlin, 1969.

[3] Forstnerič, F., and J.-P. Rosay: Localization ot the Kobayashi metric and the boundary continuity of proper holomorphic mappings. - Math. Ann. 279, 1987, 239-252.

[4] Gehring, F. W., K. HaG, and O. Martio: Quasihyperbolic geodesics in John domains. Math. Scand. 65, 1989, 75-92.

[5] Gehring, F. W., and B. G. OsGood: Uniform domains and the quasi-hyperbolic metric. - J. Anal. Math. 36, 1979, 50-74.

[6] Gilbarg, D., and N. Trudinger: Elliptic partial differential equations of second order. Springer Verlag, Berlin, 1977.

[7] Krantz, S. G., and H. R. Parks: Distance to $\mathcal{C}^{k}$ hypersurfaces. - J. Differential Equations 40, 1981, 116-120.

[8] Martin, G. J.: Quasiconformal and bi-Lipschitz homeomorphisms, uniform domains and the quasihyperbolic metric. - Trans. Amer. Math. Soc. 292, 1985, 169-191.

[9] Nikolov, N., and L. Andreev: Estimates of the Kobayashi and quasi-hyperbolic distances. - Ann. Mat. Pura Appl. 196, 2017, 43-50.

[10] Nikolov, N., M. Trybula, and L. Andreev: Boundary behavior of invariant functions on planar domains. - Complex Var. Elliptic Equ. 61, 2016, 1064-1072.

[11] Pommerenke, Ch.: Boundary behaviour of conformal maps. - Grundlehren Math. Wiss. 299, Springer, Berlin, 1992.

[12] Vuorinen, M.: Conformal geometry and quasiregular mappings. - Lecture Notes in Math. 1319, Springer, Berlin, 1988. 